\title{
Intramucosal duodenal adenocarcinoma concomitant with Saint's triad
}

\author{
Matheus Dantas Gomes Gonçalves, ${ }^{1}$ Vinicius Grigolli, ${ }^{1}$ Thereza Cristina Carvalho Kalmar, ${ }_{1}^{1}$ \\ Vitorino Modesto dos Santos, ${ }^{2}$ Lucimara Sonja Villela, ${ }^{1}$ Lister Arruda Modesto dos Santos ${ }^{1}$
}

${ }^{1}$ Advanced General Surgery, State Worker's Hospital, São Paulo-SP; ${ }^{2}$ Medicine Department, Armed Forces Hospital and Catholic University, Brasília-DF, Brazil

\begin{abstract}
Duodenal tubulovillous adenomas are rare and have malignant potential. Their successful management depends on the preoperative diagnosis and adequate excision. Endoscopic mucosal resection or submucosal dissection can control superficial tumors. Adenocarcinoma of the duodenum may originate in tubular or tubulovillous adenoma, and possible diagnostic challenges occur concerning the endoscopic biopsy findings. We report a 62 -year-old female with chronic epigastric burning and a bolus sensation. Upper gastrointestinal endoscopy showed a non-ampullary duodenal tumor, and the biopsy study diagnosed a tubulovillous adenoma. Further mucosectomy revealed an intramucosal adenocarcinoma in a tubulovillous adenoma with a tumor-free pedicle. Besides the intestinal neoplasms, the patient had the diagnosis of classical Saint's triad; and the manifestations were hiatus hernia, gallbladder disorder, and colonic diverticula. The objective is to report two scarcely described conditions and comment on controversial points of view about the concomitance of Saint's triad and malignancy. Case reports can reduce late diagnosis, enhancing the suspicion index on rare diseases.
\end{abstract}

\section{Introduction}

Duodenal adenocarcinomas are rare, represent $0.3 \%$ of gastrointestinal and $33-45 \%$ of the small

Correspondence: Vitorino Modesto dos Santos, Hospital das Forças Armadas, Estrada do Contorno do Bosque s/n, Cruzeiro Novo, 70.658-900, Brasília-DF, Brazil.

Tel.: +55.61.39662103 - Fax: +55.61.32331599.

E-mail: vitorinomodesto@gmail.com

Key words: Adenocarcinoma/diagnosis; tubulovillous adenoma; duodenal neoplasm/pathology; endoscopy/digestive system; Saint's triad.

Contributions: all authors contributed equally to the writing of the article.

Conflict of interests: the authors declare no potential conflict of interests.

Ethics approval: not required.

Informed consent: the patient gave informed consent.

Received for publication: 25 September 2020.

Revision received: 11 January 2021.

Accepted for publication: 12 January 2021.

This work is licensed under a Creative Commons Attribution NonCommercial 4.0 License (CC BY-NC 4.0).

${ }^{\circ}$ Copyright: the Author(s), 2021

Licensee PAGEPress, Italy

Italian Journal of Medicine 2021; 15:120-123

doi:10.4081/itjm.2021.1376 bowel tumors, and with nonspecific symptoms, the diagnosis is incidental. ${ }^{1-10}$ They are prevalent in the second duodenal portion of people between 40 and 60 years. ${ }^{10}$ Risk factors include alcohol, smoked or salt-cured foods, red meats, refined sugars, hereditary nonpolyposis colorectal cancer, and familial adenomatous polyposis. ${ }^{2,7,8}$ Duodenal adenocarcinoma is strongly associated with the rare tubulovillous and villous adenomas, a phenomenon favoring misdiagnosis or late cancer diagnosis. ${ }^{1,6-9}$ Endoscopy and imaging studies may detect the intraluminal polypoid changes. ${ }^{1,4,6-9}$ Minimally invasive (endoscopic mucosal resection or submucosal dissection) for the superficial and complete resection for locally advanced tumors are surgical options. ${ }^{10,11}$ Adjuvant or neoadjuvant chemotherapy are tools for patients considered inoperable. ${ }^{7,10}$ Saint's triad (ST) includes hiatus hernia, cholecystopathy, and colon diverticulosis, but aortic aneurysm and dilated cardiomyopathy are additional changes of the pentad. The term herniosis refers to the tendency of any type of hernia in individuals with ST. ${ }^{12-14}$ Interesting are some published comments about a hypothetical inverse relationship between molecular mechanisms of ST and the development of malignancies. ${ }^{12,13}$ Although ST is considered an uncommon condition, with relatively few reported cases, the authors strongly believe that it is an underdiagnosed and underreported disorder. Case reports can enhance the suspicion index of health care workers on rare diseases. 


\section{Case Report}

A 62-year-old Brazilian woman, hypertensive and with dyslipidemia, came to the hospital to evaluate burning epigastric pain and bolus sensation beginning approximately one year ago. She had no changes in the intestinal rhythm or weight loss and denied tobacco smoking and alcohol abuse. Medicines for continuous use were indapamide, spironolactone, nebivolol, acetylsalicylic acid, and simvastatin. Medical history: cesarean section, cholecystectomy (cholesterol polyps) ten years ago. ${ }^{15}$ Family antecedents: lung cancer (father), bone cancer in the foot (aunt), intestinal cancer (uncle). Three months earlier, an upper digestive endoscopy (UDE) revealed a vegetating sessile lesion contralateral to the duodenal papilla with a confirmed diagnosis of tubulovillous adenoma, then classified as group 4 of the revised Vienna consensus. The purpose of hospitalization was to perform UDE with duodenoscopy for routine control. On physical examination, she had a class II obesity (BMI: $36.2 \mathrm{~kg} / \mathrm{m}^{2}$ ), afebrile and hydrated with a globose, flabby, depressible, and painless abdomen, without visceromegaly or a palpable mass. Laboratory routine, including blood cell counts and biochemical determinations, revealed results within the normal parameters. Levels of the tumor markers carcinoembryonic antigen (CEA) and CA 19-9 were unremarkable. Computed tomography (CT) of the abdomen and pelvis showed a hypoattenuating image on the tail of the pancreas, measuring 0.5 $\mathrm{cm}$, of probable cystic nature, and colonic diverticula. The aorta was atheromatous but of normal diameter. Chest CT showed the aorta and heart with regular dimensions. The UDE with duodenoscopy detected intense distal erosive esophagitis (Grade C of Los Angeles), a sliding hiatal hernia (type 1), and moderate erosive antrum gastritis. The duodenal papilla was normal, with intact mucosa and spontaneous bile drainage. Adjacent to the papilla, there was an extensive vegetating lesion, with a pinky irregular surface, and measuring approximately 30-40 $\mathrm{mm}$. Biopsy samples revealed a tubulovillous adenoma with high-grade epithelial dysplasia. Besides colonic diverticula, the colonoscopy evaluated the distal ileum, where there were flat erosions with fibrin, and the histopathologic study showed hyperplasia of lymphoid follicles. Three months later, a UDE found a subpedunculated polyp measuring approximately $50 \mathrm{~mm}$, with an irregular surface and a wine color, in the bulbar flexure and extending to the second duodenal portion $3.0 \mathrm{~cm}$ from the major duodenal papilla (Figure 1). After the mucosectomy, the established diagnosis was of intramucosal

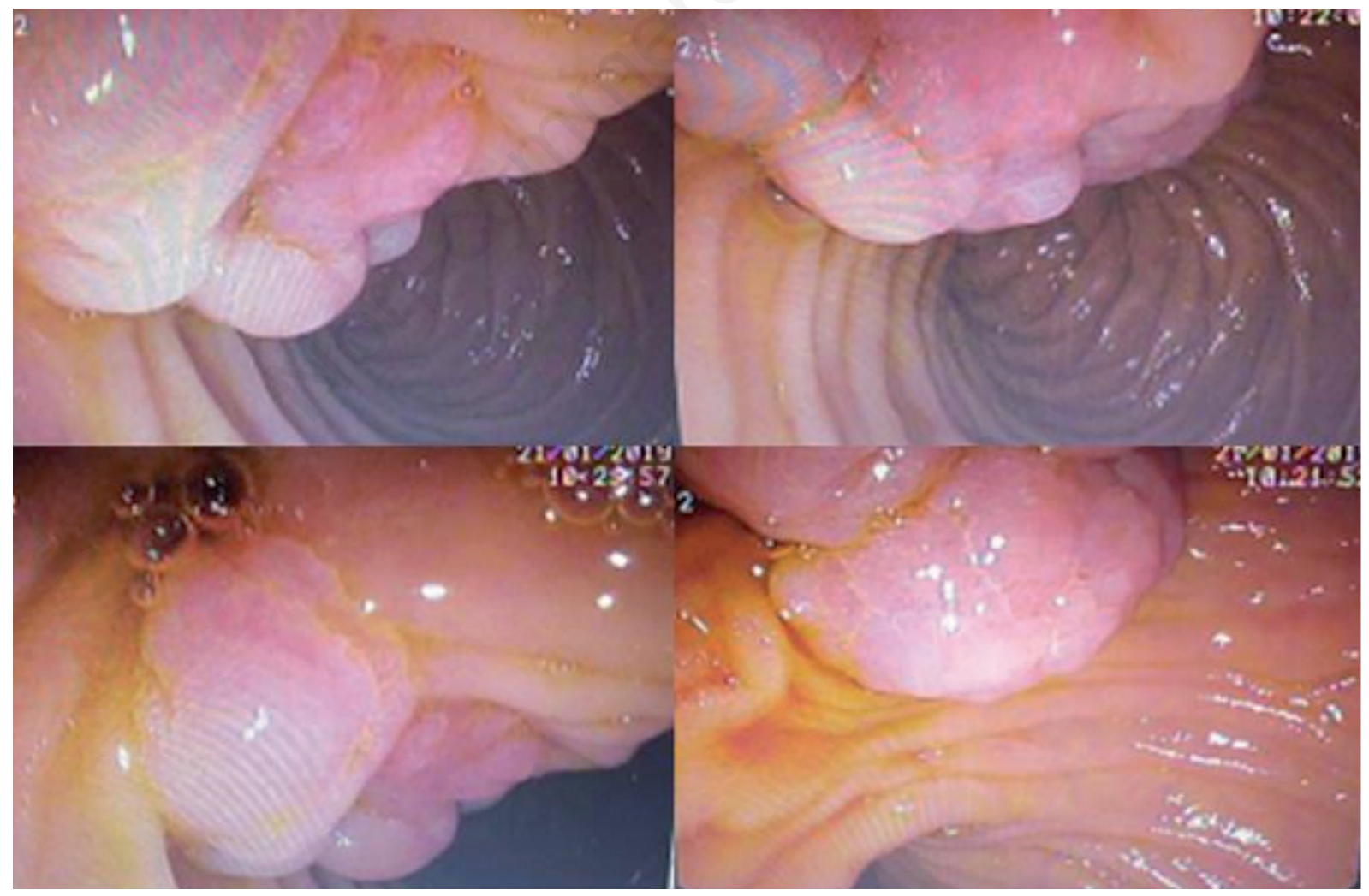

Figure 1. Upper digestive endoscopic images of the duodenal polyp with an irregular surface of a wine color, found in the bulbar flexure and extending to the second duodenal portion, approximately at $3.0 \mathrm{~cm}$ from the major duodenal papilla. 
adenocarcinoma originating from a tubulovillous adenoma and with a pedicle free of tumor (Figure 2). The asymptomatic patient remains on outpatient control with UDE every six months.

\section{Discussion}

The 62-year-old woman diagnosed with duodenal adenocarcinoma evolving within a tubulovillous polyp also had clinical and imaging features of classical ST. ${ }^{12-}$ ${ }^{14}$ The rare incidence of the tubulovillous pattern is less than $1 \%$ of all duodenal tumors. ${ }^{8}$ Duodenal adenocarcinoma represents $0.3 \%$ of gastrointestinal tumors, mainly located in the second part of the organ, affecting people between the ages of 40 and 60 years. ${ }^{10}$ As often occurs with nonspecific symptoms, her diagnosis of cancer was incidental. ${ }^{4,5,8}$ She had first abdominal studies revealing a polypoid duodenal lesion measuring 30$40 \mathrm{~mm}$, and the endoscopic biopsy was negative for malignancy. However, a major finding was the presence of high-grade epithelial dysplasia (intense atypia). Further surgical excision of the polyp revealed associated adenocarcinoma with a pedicle free of tumor. Advanced adenomas of tubulovillous or villous patterns, measuring $10 \mathrm{~mm}$ or larger, often exhibit high-grade dysplasia and have an elevated risk for malignant evolution. ${ }^{7}$ Duodenal adenomas are precursors of malignancy, as occurs in colorectal cancer, after multiple specific genetic changes that contribute to the uncontrolled growth process. It is a prolonged phenomenon by which few adenomas undergo a total transformation. ${ }^{7}$ Worthy of note in the present case study was the incidental diagnosis of the typical ST manifested by a hiatus hernia, gallbladder disorder, and colonic diverticula. ${ }^{12-14}$ According to the literature, this report involves the unsuspected, underreported, or very infrequent association of the intramucosal duodenal adenocarcinoma with the ST. ${ }^{1-15}$ Personal (duodenal polypoid lesion) and family (uncle with intestinal cancer) antecedents may have a role in the origin of the duodenal malignancy herein described. Nevertheless, there were no consistent findings to confirm the diagnostic hypothesis either of hereditary nonpolyposis colorectal cancer or familial adenomatous polyposis. Similar to previous descriptions, the histopathological pattern revealed by the initial endoscopic biopsy specimens established a benign duodenal polyp diagnosis. ${ }^{14,6}$ Therefore, a significant concern in this setting is related to the possibility of pitfalls that may propitiate diagnostic mistakes and late evidence of an associated adenocarcinoma. Diagnosis of the tumor in the early stage, as found in the present case, allows less invasive surgical procedures, including endoscopic mucosal or submucosal interventions. ${ }^{3,11}$ More advanced stages of the tumors imply more invasive management, including partial or segmental and en bloc duodenal resection; duodenal cephalo-pancreatectomy; segmental duodenojejunal resection; Whipple procedure, or another palliative care. ${ }^{1-8,10}$ Currently, the endoscopic mucosal resection and submucosal dissection remain scarcely utilized by endoscopists of the West in the treatment of superficial duodenal tumors. ${ }^{11}$ However, submucosal dissection has a good rate of efficacy and safety, and the majority of resected lesions in the upper gastrointestinal tract are intramucosal adenocarcinoma. ${ }^{11}$ An additional primary concern is about the alleged inverse mechanisms between the development of malignancies and the physiopathology of hernias in patients with ST. The relationship of the triad with malignancy stands controversial. Increased collagen I and fibroblasts in extracellular matrices of people with ST may impair cancer development as seen in the present case. ${ }^{12}$ However, ST occurred associated with a signet ring cell gas-

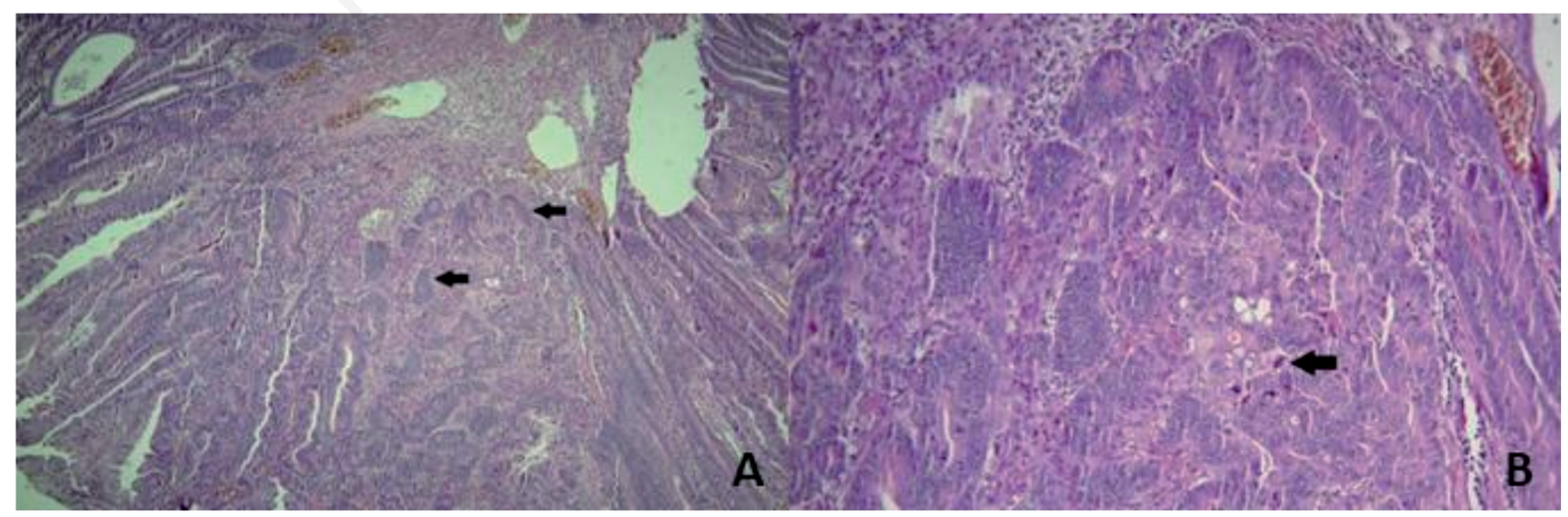

Figure 2. Photomicrographs of samples from the duodenal tumor. A) Changes of the architecture with irregular glands, nuclear stratification, hyperchromasia, and mitotic figures (arrows), compatible with intramucosal adenocarcinoma (H $\& \mathbf{E}, 4 \mathrm{x}$ ); and $\mathrm{B}$ ) Evident figures of mitosis (arrow) consistent with the malignant process of intramucosal adenocarcinoma $(H \& \mathbf{E}, 10 x)$. 
tric adenocarcinoma plus a malignant sigmoid polyp in a Brazilian patient. ${ }^{13}$ This patient was hypertensive, but the hypothesis of Saint's pentad or tetrad was ruled out by the absence of manifestations as dilated cardiomyopathy and aortic aneurism. ${ }^{12,13}$ This duodenal polyp presented accentuated atypia, but the surgical margins were free. One must better clear the hypothesis of an inverse relationship between ST and cancer.

\section{Conclusions}

This case study involves a duodenal adenocarcinoma related to a tubulovillous adenoma and concomitant with typical ST, phenomenon having a random relationship uniquely. Single case studies have an inherent weakness, but the data can merit further research.

\section{References}

1. Anand U, Pandey MK, Priyadarshi RN, et al. Large tubullo-villous adenoma of duodenum presenting as gastric outlet obstruction. J Clin Diagn Res 2015;9:PD03-4.

2. Biello AR, Lin-Hurtubise KM, Condon FJ, Allen EJ. Duodenal adenocarcinoma at the ligament of Treitz: management and outcome. Hawaii J Health Soc Welf 2019;78:316-9.

3. Hwang KL, Kim GH, Lee BE, et al. Long-term outcomes of endoscopic resection for non-ampullary duodenal epithelial tumors: A single-center experience. Turk J Gastroenterol 2020;31:49-57.

4. Jiménez-Fuertes M, Ruíz-Tóvar J, Díaz-García G, Durán-Poveda M. [Moderately differentiated adenocar- cinoma of the third duodenal portion]. Cir Cir 2017;85:76-9.

5. Khanal S, Joshi U, Bhattarai A, et al. Primary duodenal adenocarcinoma: uncommon tumor, uncommon presentation. A case report and review of the literature. Int Med Case Rep J 2020;13:165-9.

6. Lemyé AC, Guy-Viterbo V, van Vyve E. Adenocarcinoma of the duodenum arising in a tubulo-villous adenoma. Acta Chir Belg 2009;109:95-7.

7. Lubner MG, Menias CO, Johnson RJ, et al. Villous gastrointestinal tumors: multimodality imaging with histopathologic correlation. Radiographics 2018;38: 1370-84.

8. Malik MN, Shah Z, Rafae A, et al. Small intestinal tumors: a rare case of tubulovillous adenoma in duodenum. Cureus 2019;11:e4671.

9. Morotti A, Gned D, Di Martino L, et al. Small bowel cancer diagnosis: role of nuclear magnetic resonance. Ital J Med 2016;10:134-6.

10. Velandia C, Morales RD, Coello C, et al. Neoadjuvant chemotherapy in locally advanced duodenal adenocarcinoma. E Cancer Med Sci 2018;12:816.

11. Araújo-Martins M, Pimentel-Nunes P, Libânio D, et al. How is endoscopic submucosal dissection for gastrointestinal lesions being implemented? Results from an international survey. GE Port J Gastroenterol 2020;27:1-17.

12. Dos Santos VM, Dos Santos LAM. Comments on Saint's triad. Surg Case Rep 2015;1:115.

13. Santos VM, Carneiro MV, Soares VVP, et al. An elderly man with gastric cancer, Saint's triad and Heyde's syndrome. Rev Gastroenterol Peru 2018;38:289-92.

14. Santos VM, Yano VM, Alves da Cunha GLQ, et al. Yellow nail syndrome, Phrygian cap gallbladder and Saint's triad? Case report. Méd Uis 2015;28:387-91.

15. Terada T. Histopathologic features and frequency of gallbladder lesions in consecutive 540 cholecystectomies. Int J Clin Exp Pathol 2013;6:91-6. 